\title{
Subliminal conditioning of vestibular perception generalizes within otolith organs
}

\author{
Aram Keywan $^{1} \cdot$ Gharam Yassin $^{1} \cdot$ Klaus Jahn $^{1,2} \cdot$ Max Wuehr $^{1}[$
}

Received: 1 November 2021 / Revised: 12 January 2022 / Accepted: 19 January 2022 / Published online: 3 February 2022

(c) The Author(s) 2022

\section{Dear Sirs,}

Previous studies in animals and humans provide evidence that the vestibular system is capable to autoregulate its sensitivity in response to prolonged exposure to either highor low-amplitude vestibular stimuli. In an aquatic animal model, the gain of the vestibulo-ocular reflex (VOR) was shown to decrease or increase when being exposed to sustained passive oscillatory head motion at high or low amplitudes, respectively [6]. Analogous effects were demonstrated in humans, where prolonged exposure to highintensity passive motion attenuates vestibular-mediated balance responses and increases thresholds for vestibular motion perception [7]. We recently complemented the latter finding by showing that human perceptual thresholds can conversely be decreased in response to long-lasting imperceptible passive head oscillations-a phenomenon we designated as subliminal vestibular conditioning [10]. Taken together, such bi-directional adaptive re-calibrations within vestibular information processing resemble the characteristics of a homeostatic plasticity [6] that is able to counterbalance prolonged elevated or diminished neuronal activity to stabilize vestibular encoding around a certain set point of activity [14].

Subliminal conditioning of vestibular perception is likely mediated by plasticity within central vestibular networks. Surgical ablation of the cerebellum in animal models has accordingly been shown to prevent homeostatic plasticity within the VOR circuitry [6]. Other central mechanisms such as adaptive plasticity at the synapse between vestibular afferents and first-order central neurons may further mediate homeostatic adaptions in vestibular signal processing

Max Wuehr

max.wuehr@med.uni-muenchen.de

1 German Center for Vertigo and Balance Disorders, University Hospital of Munich, Munich, Germany

2 Schön Klinik Bad Aibling, Bad Aibling, Germany
[11]. Since inputs from the different vestibular end organs, i.e., otolith and semicircular canal (SCC) receptors, show considerable central convergence $[4,5,13,15]$, it is thus conceivable that the sensitizing effects of subliminal vestibular conditioning are not specific for the particular motion plane being stimulated but rather generalize over other nonstimulated structures of the vestibular periphery.

We previously tested this hypothesis by examining whether conditioning-induced sensitization of otolith-mediated perception may also affect SSC-mediated perceptual thresholds but did not find evidence for such crosstalk [10]. The purpose of this follow-up study was to examine whether alternatively a conditioning-induced crosstalk may exist between the two otolith organs. To this purpose, we tested if translational passive motion conditioning along the horizontal interaural axes, which sensitizes utricular-mediated perception, also affects saccular-mediated perception for translations along the earth-vertical axis.

Eight healthy subjects (four males, mean age $27.1 \pm 2.1$ years) that were naive to the experimental protocol participated in the study. None of the participants reported any auditory, vestibular, or cardio-vascular disorders. All were familiar with the procedures of vestibular threshold testing from previous studies and gave their written informed consent prior to the experiments. The experimental procedures were analogous to our previous study [10]. Shortly, the experiment consisted of two parts separated by a resting period of one hour. In the first part, the effects of translational passive motion conditioning along the earth-horizontal interaural axis on utricular-mediated perception was tested (Fig. 1A). In the second part, potential crosstalk between the horizontal conditioning stimulus and saccular-mediated perception for translations along the earth-vertical superior-inferior axis was tested (Fig. 1B). For both experiments, vestibular direction-recognition thresholds (DRT) at $1 \mathrm{~Hz}$ were determined immediately before (baseline), immediately after (post $0 \mathrm{~min}$ ), and $20 \mathrm{~min}$ after (post $20 \mathrm{~min}$ ) conditioning while participants were seated on 


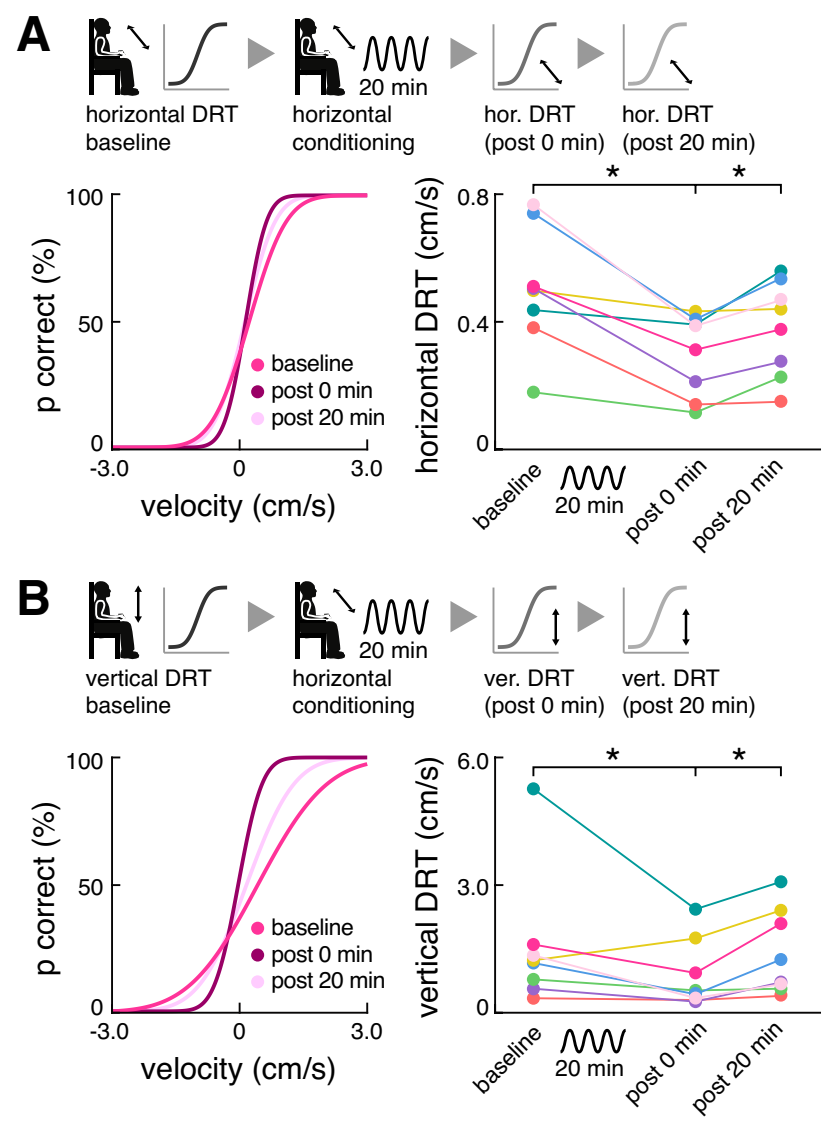

Fig. 1 Conditioning effects on horizontal and vertical vestibular perceptual thresholds. Within- and cross-plane conditioning effects were tested in two separate sessions. A Upper panel: experimental procedure for testing within-plane effects of horizontal translational conditioning on utricular-mediated horizontal translational DRTs; lower panel: exemplary psychometric curve fits (left) and group results (right) of the perceptual performance for horizontal translational motion stimuli immediately before (baseline), immediately after (post $0 \mathrm{~min}$ ), and $20 \mathrm{~min}$ after (post $20 \mathrm{~min}$ ) horizontal conditioning. B Upper panel: experimental procedure for testing cross-plane effects of horizontal translational conditioning on saccular-mediated vertical translational DRTs; lower panel: exemplary psychometric curve fits (left) and group results (right) of the perceptual performance for vertical translational motion stimuli immediately before (baseline), immediately after (post $0 \mathrm{~min}$ ), and $20 \mathrm{~min}$ after (post $20 \mathrm{~min}$ ) horizontal conditioning. DRT direction recognition threshold

a 6DOF motion platform (Moog, 6DOF2000E, East Aurora, New York; 150 trials each following a 3-down 1-up paradigm $[3,8])$. DRTs were determined in darkness with eyes closed and participants wore noise-canceling headphones to mask any sound cues resulting from the platform motion.

The subliminal conditioning stimulus consisted of a $1 \mathrm{~Hz}$ sinusoidal translational motion along the horizontal interaural axis. Conditioning was applied for a total duration of 20 min while participants were quietly sitting on the motion platform with their head stabilized in an upright, straight-ahead position and eyes closed $[6,10]$. For each participant, the conditioning stimulus amplitude was individually adjusted to $70 \%$ of their baseline vestibular perceptual threshold for horizontal translations and consequently remained below perceptual level. Statistical comparisons were performed on the log-transformed DRTs to achieve normal distribution [8]. The effects of horizontal conditioning on utricular- and saccular-mediated DRTs were evaluated by a repeated-measures ANOVA and Bonferroni post hoc analysis with the factor session (baseline, post $0 \mathrm{~min}$, post 20 min) using SPSS (version 26.0, IBM corp., Armonk, NY).

Baseline assessment of vestibular DRTs yielded an average of $0.50 \pm 0.19 \mathrm{~cm} / \mathrm{s}$ for horizontal interaural and $1.51 \pm 1.57 \mathrm{~cm} / \mathrm{s}$ for vertical translational motion. The individual conditioning stimulus was accordingly set to $70 \%$ of the baseline horizontal DRT (in average $0.35 \pm 0.13 \mathrm{~cm} / \mathrm{s}$ peak velocity) and not perceived by any of the participants. Horizontal translational conditioning significantly lowered horizontal DRTs in all participants by in average $39 \pm 19 \%$ (post 0 min vs. baseline: $\mathrm{F}_{1,7}=44.3, p=0.006$; Fig. $1 \mathrm{~A}$ ). Horizontal DRTs had returned to baseline level $20 \mathrm{~min}$ after conditioning (post $20 \mathrm{~min}$ vs. post $0 \mathrm{~min}$ : $\mathrm{F}_{1,7}=44.3$, $p=0.016$ ). Remarkably, the effects of horizontal conditioning on vertical DRTs were found to be more or less analogous. Immediately after conditioning, vertical DRTs were significantly lowered for all except one participant by in average $35 \pm 35 \%$ (post 0 min: vs. baseline: $F_{1,7}=17.2$, $p=0.041$; Fig. 1B) and had returned to baseline $20 \mathrm{~min}$ after conditioning (post $20 \mathrm{~min}$ vs. post $0 \mathrm{~min}: \mathrm{F}_{1,7}=17.2$, $p=0.016$ ).

These results first confirm our previous observation that an exposure to prolonged low-intensity oscillatory head motion can induce sensitization of the vestibular perceptual capacity in healthy adults [10]. In agreement with prior observations in animal models and humans, we found that the conditioning-induced lowering of perceptual thresholds was transient with a relatively short time constant and had reversed already $20 \mathrm{~min}$ after cessation of the conditioning stimulus. This observation further complements previous reports on analogous transient adaptions of sensitivity in human visual, auditory, and somatosensory perception induced by prolonged subliminal sensory stimulation $[1,2$, 16].

Notably, we found that passive translational motion conditioning along the horizontal interaural axis equally sensitized utricular-mediated perception with a sensory epithelium aligned to the conditioning movement and saccular-mediated perception with a sensory epithelium orthogonal to motion direction. Although we cannot exclude a small amount of co-activation of saccular afferents during utricular conditioning (due to variations in head orientation and/or undirected vibrational noise induced by the motion platform [3]), this would most likely not suffice to induce sensitizing 
effects of the same order of magnitude (threshold reduction of $39 \%$ for utricular- vs. $35 \%$ for saccular-mediated perception).

Alternatively, we propose that the generalization of conditioning effects over non-stimulated directions of motion is rather of central origin. Utricular and saccular otolith afferents are commonly tuned to respond to a particular preferred direction vector. In contrast, central vestibular nuclei that frequently receive convergent inputs from both otolith organs typically exhibit a multi-dimensional tuning and equally respond to motion along two or even three orthogonal direction vectors $[4,5]$. Hence, homeostatic plastic changes of neuronal encoding within central vestibular nuclei neurons could mediate adaptions of vestibular sensitivity for both stimulated was well as non-stimulated orthogonal axes of motion.

In contrast to our present findings, previous reports, however, indicated that conditioning-induced adaptions in vestibular sensitivity are rather specific for the plane being stimulated $[6,10]$. Accordingly, our preceding study did not provide evidence that sensitizing effects of utricular-conditioning also affect horizontal-SSC-mediated vestibular perception [10]. One reason for this apparent discrepancy could be the less pronounced central convergence pattern of utricular and horizontal SSC afferents compared to that of afferents from the two otolith organs [15]. Furthermore, prolonged horizontal passive head rotations were found to only induce homeostatic adaptions of the horizontal but not the vertical rotational VOR [6]. This observation was analogously attributed to a rather infrequent and weak convergence of afferent inputs from different SCC along the central VOR circuitry [13].

In conclusion, this study demonstrates that vestibular perception can be transiently sensitized by a prolonged subliminal stimulation with imperceptible oscillatory head motion. This effect not only targets the stimulated sensory end organ structures but may at least partly generalize over other non-stimulated receptors of the vestibular periphery. In such a way, subliminal vestibular conditioning may be used therapeutically to boost previously suggested autoregulatory processes in the vestibular system $[9,12]$ to treat decrements of vestibular sensitivity in the elderly and patients with uncompensated vestibular hypofunction. A practical implementation of subliminal vestibular conditioning in a rehabilitation context may be accomplished by for instance prolonged standing on a balance board or sitting on an exercise ball. However, since the here reported conditioninginduced changes in vestibular sensitivity were transient and receded with a relatively short time constant, further research is required to examine whether longer lasting or repeated conditioning may yield temporally extended homeostatic adaptions of vestibular sensitivity.
Acknowledgements The work was supported by the German Federal Ministry for Education and Science (BMBF, IFB 01EO1401).

Funding Open Access funding enabled and organized by Projekt DEAL.

\section{Declarations}

Conflicts of interest The authors declare that they have no conflict of interest.

Ethical standards The Ethics Committee of the Medical Faculty of the University of Munich approved the study protocol, which was conducted in conformity with the Declaration of Helsinki.

Open Access This article is licensed under a Creative Commons Attribution 4.0 International License, which permits use, sharing, adaptation, distribution and reproduction in any medium or format, as long as you give appropriate credit to the original author(s) and the source, provide a link to the Creative Commons licence, and indicate if changes were made. The images or other third party material in this article are included in the article's Creative Commons licence, unless indicated otherwise in a credit line to the material. If material is not included in the article's Creative Commons licence and your intended use is not permitted by statutory regulation or exceeds the permitted use, you will need to obtain permission directly from the copyright holder. To view a copy of this licence, visit http://creativecommons.org/licenses/by/4.0/.

\section{References}

1. Amitay S, Irwin A, Moore DR (2006) Discrimination learning induced by training with identical stimuli. Nat Neurosci 9:1446-1448

2. Bliem B, Muller-Dahlhaus JF, Dinse HR, Ziemann U (2008) Homeostatic metaplasticity in the human somatosensory cortex. J Cogn Neurosci 20:1517-1528

3. Chaudhuri SE, Karmali F, Merfeld DM (2013) Whole body motion-detection tasks can yield much lower thresholds than direction-recognition tasks: implications for the role of vibration. J Neurophysiol 110:2764-2772

4. Chen-Huang C, Peterson BW (2006) Three dimensional spatialtemporal convergence of otolith related signals in vestibular only neurons in squirrel monkeys. Exp Brain Res 168:410-426

5. Dickman JD, Angelaki DE (2002) Vestibular convergence patterns in vestibular nuclei neurons of alert primates. J Neurophysiol 88:3518-3533

6. Dietrich H, Straka H (2016) Prolonged vestibular stimulation induces homeostatic plasticity of the vestibulo-ocular reflex in larval Xenopus laevis. Eur J Neurosci 44:1787-1796

7. Fitzpatrick RC, Watson SRD (2015) Passive motion reduces vestibular balance and perceptual responses. J Physiol 593:2389-2398

8. Grabherr L, Nicoucar K, Mast FW, Merfeld DM (2008) Vestibular thresholds for yaw rotation about an earth-vertical axis as a function of frequency. Exp Brain Res 186:677-681

9. Jahn K, Naessl A, Schneider E, Strupp M, Brandt T, Dieterich M (2003) Inverse U-shaped curve for age dependency of torsional eye movement responses to galvanic vestibular stimulation. Brain 126:1579-1589

10. Keywan A, Dietrich H, Wuehr M (2020) Subliminal passive motion stimulation improves vestibular perception. Neuroscience 441:1-7 
11. Mitchell DE, Della Santina CC, Cullen KE (2016) Plasticity within non-cerebellar pathways rapidly shapes motor performance in vivo. Nat Commun 7:11238

12. Peters RM, Blouin JS, Dalton BH, Inglis JT (2016) Older adults demonstrate superior vestibular perception for virtual rotations. Exp Gerontol 82:50-57

13. Straka H, Dieringer N (2004) Basic organization principles of the VOR: lessons from frogs. Prog Neurobiol 73:259-309
14. Turrigiano G (2011) Too many cooks? Intrinsic and synaptic homeostatic mechanisms in cortical circuit refinement. Annu Rev Neurosci 34:89-103

15. Uchino Y, Sasaki M, Sato H, Bai R, Kawamoto E (2005) Otolith and canal integration on single vestibular neurons in cats. Exp Brain Res 164:271-285

16. Watanabe T, Náñez JE, Sasaki Y (2001) Perceptual learning without perception. Nature 413:844-848 